\title{
FDA-Approved (Bremelanotide, Flibanserin) and Off-Label Medications (Testosterone, Sildenafil) to Enhance Sexual Desire/Function in Women
}

\author{
Barbara Bartlik ${ }^{1 *}$, Allison Sugarman ${ }^{2}$, Sonia Seenaraine ${ }^{3}$ and Samuel Green ${ }^{4}$ \\ Northwell Health, Zucker School of Medicine at Hofstra/Northwell, United States of America \\ ${ }^{2}$ Masters in Public Policy Candidate, Department of Psychology, University of Maryland, United States of America \\ ${ }^{3}$ Psychology Candidate, Hunter College, United States of America \\ ${ }^{4}$ Samuel Green, BaSc Candidate, Columbia University, United States of America
}

${ }^{1}$ Department of Psychiatry, Weill Cornell Medical College, Cornell University, New York Presbyterian/Weill Cornell Medical Center and Lenox Hill Hospital/

*Corresponding author: Barbara Bartlik, Department of Psychiatry, Weill Cornell Medical College, Cornell University, New York Presbyterian/Weill Cornell Medical Center and Lenox Hill Hospital/Northwell Health, Zucker School of Medicine at Hofstra/Northwell, United States of America.

Received Date: January 21, 2020

Published Date: April 30, 2020

\begin{abstract}
Bremelanotide has been available off-label through compounding pharmacies for many years for men and women seeking to improve libido. In 2019, the FDA approved use of bremelanotide (Vyleesi) for premenopausal women with hypoactive sexual desire disorder (HSDD), the second medication approved for this purpose. The first was flibanserin (Addyi), a pill to be taken daily at bedtime, and not within 2 hours of consuming alcohol. Though HSDD is highly prevalent and affects nearly six million premenopausal women, the availability of approved pharmaceuticals to treat it is relatively recent. Bremelanotide activates melanocortin receptors and pathways in the brain involved in the sexual response. Studies have shown that it improves sexual desire and lowers distress surrounding sexual interaction. Though not approved for men, bremelanotide has the potential to benefit men with low libido and erectile difficulties. As with many medications, bremelanotide is associated with adverse effects, which include nausea, flushing, injection site reactions, headache, vomiting, hypertension, and irreversible hyper-pigmentation. In addition, the offlabel prescription of testosterone in women is widespread and been used to improve libido, genitourinary symptoms, and vitality, particularly in the anti-aging community. Other pharmaceuticals that are prescribed off-label to aid female sexual function include the PDE5 inhibitors sildenafil, vardenafil, and tadalafil. Non-pharmacologic interventions for improving sexual function include sex-positive vitamins, minerals, herbs, and lifestyle changes. Combinations of the above pharmaceuticals and natural therapies may enhance efficacy while minimizing side effects. The recent approval of bremelanotide is significant as it should advance knowledge and research on female sexual dysfunction, which is a relatively neglected area. The prescription of off- label and/or compounded medications for sexual dysfunction is generally considered within the domain of integrative, functional, or complementary and alternative medicine.
\end{abstract}

Keywords: Bremelanotide; Flibanserin, libido; Hypoactive sexual desire disorder (HSDD); Sexual disorders; Sildenafil; PDE5; Women's sexuality; Off- label; Compounding; Testosterone; Integrative complementary and alternative medicine

Abbreviations: HSDD: Hypoactive Sexual Desire Disorder; FDA: Food and Drug Administration; ICD10: International Statistical Classification of Diseases and Related Health Problems; WHO: World Health Organization; PDE5: Phosphodiesterase type 5

\section{Introduction}

Bremelanotide has been prescribed, off-label, by integrative/ functional medicine practitioners for many years for both men and women who want to boostlevels of sexual desire, arousal, and orgasm. Prior to the recent approval of bremelanotide (Vyleesi @) Palatin Technologies by the Food and Drug Administration (FDA), both men and women were equally likely to use this medication for the treatment of sexualproblems[1].In2019, theFDAapproved theuse of bremelanotide for premenopausal women with hypoactive sexual desire disorder (HSDD) [2]. This is the second medication approved for this diagnosis, the first being flibanserin (Addyi ${ }^{\circledR}$ ), which was approved for acquired, generalized HSDD in premenopausal women in 2015 [3].

HSDD is the ICD10 (International Statistical Classification of Diseases and Related Health Problems, World Health Organization 
[WHO]) diagnosis to describe low libido both in men and women. In 2012, the FDA identified female sexual dysfunction as one of 20 disease areas of high priority [4]. Nearly six million premenopausal women suffer from HSDD symptoms, which include avoiding intimacy, low self-esteem, and relationship strain $[2,5,6]$.

These two FDA-approved pharmaceuticals, bremelanotide and flibanserin, do not claim to address the ability to become aroused or achieve orgasm, but rather to improve the level of sexual desire. They both work by changing brain chemistry to improve the desire for sex in premenopausal women. They are not approved for use by men or postmenopausal women, though it is reasonable to speculate that they may have a beneficial effect in those populations as well [7]. In fact, the compounding pharmacist consulted here stated that he receives as many bremelanotide orders for men with erectile disorder as he does for women and sales have been brisk for several years [1].

Until the approval of bremelanotide, flibanserin was the only FDA-approved pharmaceutical designed to treat HSDD. It is a non-hormonal, $100 \mathrm{mg}$ pill that should be taken daily at bedtime. Though effective, it was slow to gain popularity because of a contraindication against the use of any alcohol and a requirement that both the physician and the pharmacist participate in an Elements to Assure Safe Use (ETASU) Program to assure that the patient was aware that they could not drink alcohol while on this medication. However, in 2019, after new studies were considered, the contraindication against alcohol and ETASU requirements were lifted. Now, patients are to be advised that flibanserin should not be taken within two hours of consuming alcohol due to the risk of hypotension and syncope and it should not be taken any evening during which three or more alcoholic beverages are consumed [3]. Flibanserin works by affecting neurotransmitters in the brain and is an agonist of the serotonin $1 \mathrm{~A}$ receptor and antagonist of the serotonin $2 \mathrm{~A}$ receptor [8]. Flibanserin has been shown to increase the number of satisfying sexual experiences, increase sexual desire, and reduce associated distress [8].

In contrast, bremelanotide is used on an as-needed basis prior to sexual activity, and alcohol consumption is not restricted with its use [9]. Bremelanotide works through a different mechanism of action than flibanserin and helps to activate pathways in the brain involved in the body's normal sexual response [9]. It stimulates melanocortin receptors, which are involved in the regulation of mood, thinking, and pigmentation; these receptors may promote appetite-suppressant (anorectic) and aphrodisiac effects, among others [7]. The package insert for bremelanotide states that 1.75 $\mathrm{mg} / 0.3 \mathrm{ml}$ should be injected subcutaneously into the thigh or abdomen 45 minutes prior to sexual activity [9]. This results in rapid and high bioavailability which declines over the following several hours. As dispensed by the compounding pharmacist consulted for this review, an initial dose of $1 \mathrm{mg}$ is usually prescribed, which may be increased to a maximum of $2 \mathrm{mg}$, if desired [1]. The low initial dose reduces the risk of nausea [1].
Studies have shown that bremelanotide improves selfreported feelings of desire and lowers distress surrounding sexual interaction in premenopausal women with HSDD. However, it does not enhance sexual performance or affect the frequency of satisfying sexual events [9].

As with many medications that offer worthwhile benefits, bremelanotide (Vylessi) is associated with adverse effects. Nausea is the most frequent by far (40\%), but it usually improves with the second dose, and decreases to $3 \%$ after subsequent doses [9]. In studies, $13 \%$ of patients taking bremelanotide received antiemetic therapy. Eight percent discontinued participation due to nausea [9]. Other adverse effects include flushing, injection site reactions, headache, a transient increase in blood pressure, and vomiting. Patients who experienced a transient increase in blood pressure (a maximum of $6 \mathrm{~mm} \mathrm{Hg}$ systolic and $3 \mathrm{~mm} \mathrm{Hg}$ diastolic peaking in 24 hours) also experienced a decrease in heart rate of up to five beats per minute. These changes were gone within 12 hours after the injection. To minimize the possibility of pronounced blood pressure changes, patients should take no more than one dose in 24 hours [9]. Because of the cardiovascular effects, bremelanotide is contraindicated in patients with uncontrolled hypertension or known cardiovascular disease.

Hyperpigmentation may also occur especially if bremelanotide is used often. To minimize the risk of hyperpigmentation, patients should not take more than eight doses of bremelanotide per month [9]. Focal hyperpigmentation has been reported by $1 \%$ of patients who received up to eight doses per month. The discoloration may not resolve upon stopping its use, and may occur on the face, breasts, gingiva and elsewhere [9].

Testosterone is generally believed to be the hormone most closely associated with sexual desire in both men and women [10]. Though there are many FDA-approved testosterone preparations for men, none are approved for use in women. Attempts to gain approval in the United States for a testosterone gel and patch for women were not successful [10]. In the case of the patch, long term data on safety were considered inadequate and in the case of the gel, efficacy in secondary endpoints failed to achieve significance. However, in Europe, Canada, Australia, and the United Kingdom, various forms of testosterone therapy for women are approved. In the United States off-label prescription of testosterone for women is widespread and increasing [11], despite some risks, particularly among those in the anti-aging community to address libido, urogenital symptoms, menopausal symptoms, muscle strength, and energy level.

Typically, off-label testosterone therapy for women is prescribed trans-dermally in dosages of 0.5 - $2 \mathrm{mg}$ daily [10], or less frequently. In addition, vaginal testosterone preparations may be applied directly to the clitoris and vulva. Testosterone levels should be monitored and maintained in the normal range (15-70 $\mathrm{ng} / \mathrm{dl}$ ) [10]. Patients should be monitored for adverse effects, such 
as hirsutism, deepening of the voice, male pattern hair loss, and clitoral enlargement. Since testosterone is a precursor to estradiol, it should not be prescribed for women with estrogen sensitive cancers.

It is important to ensure that hormones are optimized in both genders, and that women with sexual dysfunction are not experiencing vaginal discomfort due to a lack of estrogen. If so, they may need estradiol supplementation with or without progesterone, if not contraindicated due to a history of cancer.

The PDE5 inhibitors including sildenafil (Viagra®Pfizer), tadalafil (Cialis®Lilly), and vardenafil (Levitra®Bayer), are, as a class, the world's most prescribed type of medication for a range of sexual disorders in men [12]. However, the prescriptive use of PDE5 inhibitor medications for women, while promising, is not FDA-approved, nor are they strongly indicated. In women, the PDE5 inhibitors have been shown clinically to have less of an impact, yet they are promising enough to be considered an option for prescribers. Early clinical trials on the use of sildenafil in women with HSDD demonstrated that sildenafil improved the ability to, "achieve orgasm and state of arousal" [13], and showed, "significantly improved clitoral blood flow in postmenopausal women with orgasmic dysfunction" [13], but converse results were also obtained in other early stage clinical studies [13]. Certainly, the efficacy and safety of PDE5 inhibitors in the treatment of HSDD and other sexual disorders in women requires further study [14]. Nonetheless, some providers have prescribed PDE5 inhibitor medications in an off-label manner with good results. These medications should be considered as a possible treatment option for women with sexual dysfunction.

Non-pharmacological interventions for improving women's and men's sexual function can include cutting back on medications that cause sexual side effects and supplementing with certain sex positive vitamins, minerals, and herbs $[15,16]$. A new holistic approach to healing sexual dysfunctions via multiple practices is being developed by diverse expert integrative practitioners [17]. This integrative and functional medicine approach includes targeted, evidence-based use of botanicals and supplements, improving macro-nutrition toward the Mediterranean diet pattern, and lifestyle changes such as increased exercise and better sleep. Hormone balancing, weight loss, and metabolic remediation also may be needed, as these are implicated in some sexual dysfunctions, such as those caused by diabetes and obesity. This approach holds great promise and has the added value of addressing patients' sexual concerns to increase patient-practitioner alliance, increase understanding of patients' mental health status, and improve their engagement with general and mental health-supporting behaviors.

\section{Discussion}

Bremelanotide used to augment sexual function has long been available off-label to men and women of all ages through compounding pharmacists. Now it is approved for use only in premenopausal women with HSDD. Flibanserin is approved for use in the same population. Men, postmenopausal women, and premenopausal women with sexual performance issues may also benefit from treatment with these medications but their use in these populations is not approved.

It is possible that the demand among men for off-label use of bremelanotide will increase now that the medication has achieved approval for premenopausal women with HSDD. Men may also show interest in the off-label use of flibanserin, since the contraindication against alcohol and other restrictions were lifted last year. These may provide more options for men, particularly those who can't or do not want to take PDE5 inhibitors or testosterone. The increased visibility of bremelanotide and flibanserin may similarly lead to greater interest in their use by postmenopausal women and women with other types of sexual disorders such as problems with arousal and orgasm. More studies are needed on the use of flibanserin and bremelanotide in different populations. As sexual medicine research is relatively underfunded, clinical feedback from off-label use is likely to be disseminated first.

One drawback of these compounded and/or off-label medications is that they can be expensive and are not covered by insurance or discount offerings. Physicians are also reluctant to prescribe medications that are not FDA-approved, and often refrain from doing so unless prompted by the patient.

There may be a synergistic effect among the above-mentioned medications - bremelanotide, flibanserin, testosterone, and PDE5 inhibitors. Patients may want to take two or more in combination. Sometimes taking low dosages of more than one medication can minimize side effects while optimizing therapeutic effects. Studies on the efficacy and safety of combined use of these and other sexually enhancing medications, as well as of integrative medicine interventions, in women and men, are needed. Doctors will continue to have many unanswered questions about combinations of sexually enhancing medications in response to their patients' requests and needs. They should also become conversant with the diverse causes of sexual dysfunction and with the integrative and functional medicine options to address them.

Sexual difficulties often are at the intersection of the biological, psychological, and behavioral, and often are an indicator of other medical and psychiatric issues. As such, they deserve to be taken more seriously by all physicians. An integrative approach and wider medical interest can lead to providing more and better pharmaceutical and integrative solutions to improve sexual health in both women and men.

\section{Conclusion}

HSDD is a pervasive problem seen in the field of sexual health, affecting the lives of millions of patients, particularly women. It has real-life and life-long consequences. The FDA-approval of bremelanotide and the relaxation of restrictions on flibanserin in 2019 may stimulate further research and advance knowledge 
regarding HSDD, and other sexual disorders in women. These are relatively neglected areas of research, because they are not considered a medical priority and are not life threatening. Nonetheless, they cause much suffering for patients and their partners and can lead to the dissolution of otherwise healthy relationships, with a cascade of consequences for individuals and families. In addition to these promising pharmaceuticals, other integrative approaches should be tried and studied.

The authors hope this paper sheds light on the fact that patients are using several off-label prescription medications to enhance sexual functioning. These include bremelanotide for men and postmenopausal women, testosterone for women, and PDE5 inhibitors for women. This situation has come about due to a lack of approved treatments for sexual disorders outside of a narrow area of focus.

\section{Acknowledgment}

We wish to acknowledge Janet Mindes, Ph.D. for her scientific expertise and excellent editorial assistance.

\section{Conflict of Interest}

The authors declare no conflicts of interest.

\section{References}

1. Personal communication (2020) Nick Kirkpatrick, Tailor Made Compounding. 200 Moore Drive Nicholasville, KY, US, January 20, 2020.

2. Thomas K (2019) New Sex Drug for Women to Improve Low Libido Is Approved by the FDA. The New York Times, USA.

3. (2019) Office of the Commissioner. FDA Orders Important Safety Labeling Changes for Addyi: Studies Confirm Need for Alcohol Warnings Related to Alcohol Use with Addyi US Food and Drug Administration. April 11, 2019.

4. (2019) Office of the Commissioner. FDA Approves New Treatment for Hypoactive Sexual Desire Disorder in Premenopausal Women. U.S. Food and Drug Administration, June 21, 2019.
5. (2018) What Is Hypoactive Sexual Desire Disorder (HSDD) in Women? What Causes It? ISSM.

6. Kingsberg SA, Clayton AH, Portman D, Williams LA, Krop J, et al. (2019) Bremelanotide for the Treatment of Hypoactive Sexual Desire Disorder: Two Randomized Phase 3 Trials. Obstet Gynecol 134(5): 899-908.

7. King SH, Mayorov AV, Balse-Srinivasan P, Hruby VJ, Vanderah TW, et al. (2007) Melanocortin receptors, melanotropic peptides and penile erection. Curr Top Med Chem 7 (11): 1098-1106.

8. (2020) HCP-Addyi (Flibanserin). Pharmacological Information for Prescribers, Sprout Pharmaceuticals, Inc, US.

9. (2019) Highlights of Prescribing Information: Vyleesi (Bremelanotide Injection), for Subcutaneous Use. AMAG Pharmaceuticals, Inc, The United States Food and Drug Administration.

10. Wickman JM (2014) Androgen Therapy in Women. US Pharmacist. The Leading Journal in Pharmacy.

11. Snabes MC, Simes SM (2009) Approved Hormonal Treatments for HSDD: An Unmet Medical Need. J Sex Med 6(7): 1846-1849.

12. (2014) Which Drug for Erectile Dysfunction? Harvard Health Publishing, USA.

13. Allahdadi KJ, Tostes RC, Webb RC (2009) Female Sexual Dysfunction: Therapeutic Options and Experimental Challenges. Cardiovasc Hematol Agents Med Chem 7(4): 260-269.

14. Lo Monte G, Graziano A, Piva I, Marci R (2014) Women Taking The "Blue Pill" (Sildenafil Citrate): Such A Big Deal? Drug Des Devel Ther 8: 22512254.

15. Bartlik B, Mindes J, Haas S (2018) Sexual Dysfunction Secondary to Micronutrient Deficiency: A Common but Unrecognized Adverse Effect of Many Pharmaceuticals, Chapter 8 In: Bartlik B, Espinosa G, Mindes J, Integrative Sexual Health, Weil Integrative Medicine Library, Oxford University Press, New York, USA.

16. Yarnell E (2018) Botanicals (Adaptogens and Others) that Support or Stimulate Sexual Desire: Traditions and Science, Chapter 23 In: Bartlik B, Espinosa G, Mindes J, Integrative Sexual Health, Weil Integrative Medicine Library. Oxford University Press, New York, USA.

17. Bartlik B, Espinosa G, Mindes J (2018) Integrative Sexual Health, Weil Integrative Medicine Library, Oxford University Press, New York, USA. 